\title{
Avaliação e monitoramento da política de assistência social: uma proposta em construção
}

\author{
Nayara Cristina Bueno \\ Universidade Estadual do Paraná (Unespar)
}

\author{
Cássia Maria Carloto \\ Universidade Estadual de Londrina (UEL)
}

\begin{abstract}
Avaliação e monitoramento da política de assistência social: uma proposta em construção
Resumo: Este artigo tem como objetivo debater aspectos relacionados ao processo de implantação de sistemas de monitoramento e avaliação do Sistema Único de Assistência Social. Para isso, foi realizada pesquisa qualitativa sobre o processo piloto de monitoramento, avaliação e habilitação municipal desenvolvido no Estado do Paraná. Num primeiro momento, o trabalho discute a concepção mais difundida sobre o tema, avaliação e monitoramento. Em seguida, trata do marco legal da política de assistência social e do processo de institucionalização da sua avaliação e de seu monitoramento. Por fim, apresenta algumas considerações a partir dos dados coletados na pesquisa de campo.
\end{abstract}

Palavras-chave: Política Social. Avaliação e Monitoramento. Política de Assistência Social.

\section{Evaluation and Monitoring of Social Assistance Policy: a proposal under construction}

Abstract: This article debates aspects related to the process of implementing monitoring and evaluating systems for the Single Social Assistance System. A qualitative study was conducted about the pilot process for monitoring, evaluation and approval of municipal systems in Paraná State. The study first discusses the most broadly presented concepts about the issue of evaluation and monitoring. It then discusses the legal framework for the social assistance policy and the process of institutionalization of its evaluation and monitoring. It then presents some considerations based on data collected in the field research.

Keywords: Social Policy. Evaluation and Monitoring. Social Assistance Policy. 


\section{Introdução}

A realidade brasileira no campo da avaliação e monitoramento de políticas públicas e, especificamente, de políticas sociais possui muitas particularidades. A incompletude das reformas administrativas do Estado é uma delas e, apesar das tentativas de instaurar modelos de gestão descentralizados e participativos, o patrimonialismo continua presente nas relações sociais e o Estado brasileiro permanece burocrático e centralizador. Ademais, quando o país avança no fortalecimento da democracia e participação social nas políticas públicas, passa a sofrer influências de medidas neoliberais, repercutindo na gestão pública democrática, devido as fortes características do modelo gerencial. Este modelo de gestão presente no Estado brasileiro terá grande importância na institucionalização da função avaliação, assim como nos usos de seus resultados.

No Brasil, segundo Fagundes e Moura (2009), até os anos 1970 ocorriam avaliações sistemáticas somente nas áreas de saúde e educação. Estes estudos se restringiam a medir o alcance das metas e a adequar os meios aos objetivos dos programas. Nas demais áreas das políticas sociais, a avaliação era descontínua, pontual e, muitas vezes, não era exigida. Este cenário começou a mudar na década de 1980 devido à conjuntura econômica, política e social.

Na década de 1980, a avaliação de políticas sociais, ganha relevância em função da conjuntura socioeconômica, em que o agravamento das expressões da questão social e a escassez de recursos exigiam políticas sociais mais eficientes. Somam-se a isso as reivindicações dos movimentos sociais por políticas universalizantes e o debate sobre a participação da sociedade civil nas discussões e decisões políticas. Além disso, a expansão da avaliação também se deu porque os organismos internacionais de financiamento, como o Banco Mundial, passaram a exigi-la como condição para o repasse de recursos, impondo, a partir de então, os clássicos termos de eficiência, eficácia e efetividade.

Neste cenário, segundo Gomes (2001), a avaliação ganha impulso a partir de dois níveis: o econômico, com a finalidade de contribuir na procura de soluções que permitam aumentar a rentabilidade na aplicação de recursos; e o da redemocratização, na qual a avaliação estaria relacionada à necessidade de produzir informações para instrumentalizar a população na luta pela cidadania, em uma perspectiva política.

As experiências iniciais brasileiras, de acordo com Gomes (2001) tiveram grande influência da perspectiva tecnicista norte-americana, que se baseava no funcionalismo clássico, possuindo uma preocupação excessiva com instrumentais técnicos e metodológicos com claro viés positivista. Neste sentido, essa abordagem ignora a política social como uma intervenção na realidade, que envolve diferente sujeitos, condicionados por interesses e expectativas. Para a autora, é necessário ultrapassar este modelo estritamente econômico (noção de rentabilidade na aplicação dos recursos) e técnico da avaliação que escamoteia os princípios políticos, (sem negar a importância da técnica), e assim torná-la instrumento da população na luta pela efetivação e universalização de direitos.

As demandas de democratização da sociedade e do Estado explicitadas pelos movimentos sociais e sindicalistas na década de 1980, assim como a recusa do autoritarismo, da centralização e do caráter burocrático, clientelista e excludente das políticas sociais, significaram uma redefinição dos critérios de avaliação da política social. Segundo Melo (2009), no que se refere à engenharia político institucional, na década de 1980, são apontados para a superação da ineficácia das políticas sociais a participação dos setores excluídos na arena decisória das políticas, assim como a transparência e a descentralização. Com isso, foi introduzida na agenda pública a valorização da democracia e com ela a participação como necessária para o aperfeiçoamento do modus operandi das políticas e para torná-las mais eficientes. Assim, a avaliação é vista como instrumento de produção e divulgação de informações, que capacitam o cidadão ao exercício de controle sobre as ações do governo.

Do mesmo modo, Vaitsman e Paes-Sousa (2011) comentam que, no Brasil, a institucionalização da avaliação é parte de um processo mais amplo de construção da democracia e profissionalização da administração pública, que foi se estabelecendo a partir da Constituição Federal de 1988. Estes mecanismos de avaliação e monitoramento, ainda que representem a necessidade de controle e accountability, respondem também à pressão da sociedade por transparência e responsabilização da gestão pública.

Apesar disso, a administração pública brasileira não desenvolveu ao longo do tempo uma cultura de avaliação das políticas e programas sociais. Além de privilegiar formas de controle econômico e financeiro, ou seja, ser restrita e desenvolvida como controle de gastos, se configurou como atividade de caráter fiscalizatória, com utilização de enfoques econométricos de natureza quantitativa e, ainda, persistiu a resistência dos órgãos governamentais para a sua realização.

Ademais, quando consideramos que historicamente as políticas e programas sociais se caracterizaram como seletivas, excludentes e fragmentadas, compreendemos a importância da avaliação no seu gerenciamento. Porém, os estudos não podem se limitar a dados quantitativos, devem também privilegiar estudos qualitativos, 
preocupados com a qualidade dos serviços, programas e projetos e, sobretudo, com os impactos e as mudanças sociais nas condições de vida da população.

Como já assinalado, a administração pública brasileira carrega traços de uma cultura autoritária, burocrática e patrimonial que tem se caracterizado como obstáculo para a efetivação da avaliação. Lobo (2009) aponta que há uma postura comum na administração pública brasileira de entender a avaliação como uma atividade fiscalizatória, quase policialesca, e não como possibilidade de avançar no controle social dos programas sociais. Para o autor, há um constrangimento visível e explícito das autoridades públicas no que se refere à investigação e avaliação dos programas. As autoridades sentem-se ameaçadas quanto à perda de poder ao serem divulgados práticas indesejáveis, resultados inexpressivos ou gastos desnecessários. Com isto, as resistências à realização da avaliação se expressam na falta de apoio financeiro, político e institucional para os encaminhamentos necessários.

Jannuzzi (2011) demonstra que as dificuldades de monitoramento e avaliação no Brasil e as frustrações decorrentes disso são consequências, em boa parte, de modelos e prescrições particulares e padronizadas que não consideram o estágio do programa e o contexto de cada intervenção, ou seja, as particularidades históricas, sociais e políticas do local em que os programas estão sendo implementados. O autor também menciona a falta de análise prévia do grau de avaliabilidade dos programas e a falta de compreensão de que estas atividades se integram a processos mais amplos de gestão do programa (planejamento, orçamento, controle etc.). Também assinala que lacunas de formação e pouco domínio de conceitos e técnicas entre os gestores podem levar a frustrações com resultados e questionamentos sobre as utilidades destes instrumentos e, ainda, a supervalorização de algumas técnicas de abordagem meramente disciplinar das pesquisas aplicadas no campo.

Segundo Jannuzzi (2011), estas dificuldades contribuem para o estágio ainda incipiente de avaliação e monitoramento no Brasil, tanto nas estruturas públicas de administração das políticas, quanto nas universidades e nos centros de pesquisa. Enquanto nos Estados Unidos e países europeus a cultura de avaliação já está madura, com equipes profissionais multidisciplinares e instituições especializadas, no Brasil há muito que avançar. Aqui, parte das pesquisas são realizadas por profissionais de centros de pesquisa e universidades e, em que pesem o conhecimento teórico e técnico, em alguns casos falta conhecimento empírico da implementação de políticas sociais, o que pode gerar resultados e recomendações inexpressivos.

Não se trata apenas de introduzir conhecimentos, práticas e capacidades de avaliação de políticas públicas no Brasil, mas de mudar uma cultura, de modificar a forma como estas políticas são administradas. Joppert (2011) relaciona a fragilidade dos estudos avaliativos com a precariedade das práticas de gestão para os resultados, com as dificuldades da sociedade no exercício de pressões cidadãs por maior transparência e responsabilização e à falta de adequada instrumentação metodológica. Isto resulta de uma questão anterior que consiste na falta de formulação de estratégias, planos e programas a partir de métodos e evidências que embasem a gestão das organizações em geral, o que significa que o setor público está habituado a executar ações e planos emergenciais.

Nos últimos anos, tanto os governantes têm reconhecido a necessidade de avaliar e monitorar as políticas públicas, como também a sociedade civil tem percebido estes instrumentos que podem facilitar a transparência e a responsabilização. Com isso, em que pesem as dificuldades na realização das pesquisas avaliativas, a institucionalização destas atividades vem ganhando força.

A institucionalização, no governo federal, avança mais rapidamente, principalmente nos Ministérios de Educação (MEC), de Saúde (MS) e de Desenvolvimento Social e Combate à Fome (MDS), os quais concentram esforços em duas pontas: na produção e organização de dados mais específicos para área setorial da Política, e na condução de pesquisas avaliativas de resultados e impactos dos programas. Em outras esferas governamentais, os esforços concentram-se na elaboração de diagnósticos e no desenho de iniciativas e/ou implementação de ações previstas nos programas, sem necessariamente conter especificações de instrumentos de monitoramento e avaliação (JANUZZI, 2011). É nesse contexto que tem início o processo de institucionalização da avaliação e do monitoramento da Política de Assistência Social.

\section{A institucionalização da avaliação e do monitoramento da política de assistência social}

A informação, o monitoramento e a avaliação na gestão da assistência social estão previstos na Política Nacional de Assistência Social de 2004. Nesta regulamentação consta que a formulação e a implementação de um sistema de monitoramento e a avaliação e um sistema de informação na assistência social são ferramentas urgentes e essenciais para a consolidação da Política Nacional de Assistência Social (PNAS) e a implementação do Sistema Único de Assistência Social (SUAS), sendo esta uma construção prioritária e coletiva, pois envolve esforços dos três entes federados. 
As deliberações sucessivas, desde a I Conferência Nacional de Assistência Social de 1995 à Conferência realizada em 2003, partem do pressuposto de que a elaboração e a implementação destes instrumentos devem possibilitar "a mensuração da eficiência e da eficácia das ações previstas nos Planos de Assistência social, a transparência, o acompanhamento, a avaliação do sistema e a realização de estudos, pesquisas e diagnósticos". Com isso, pretende-se que as políticas articuladas e permanentes de informação, monitoramento e avaliação promovam novos patamares de desenvolvimento da política de assistência social, das ações realizadas e a utilização de recursos favorecendo a participação, o controle social e uma gestão otimizada da política (BRASIL, 2005, p. 70). Isso também envolve a utilização da tecnologia da informação que resulte em produção de informações e conhecimentos para os gestores, conselheiros, usuários, trabalhadores e entidades, assim como a garantia de novos espaços e patamares para a realização do controle social em níveis de eficiência e qualidade mensuráveis, através de indicadores. Tudo para que incida em um real avanço da política de assistência social para a população usuária, que é o produto esperado com o novo ideário a ser inaugurado neste campo específico.

Apesar da legislação da assistência social ter acompanhado o movimento de modernização da administração pública, pode-se afirmar que na prática os instrumentos de gestão ainda são percebidos a partir de visão técnicaoperacional e sob a perspectiva instrumental de aprimoramento da política, programas, de projetos e de benefícios.

A legislação na área da assistência social se estruturou tendo como um de seus pressupostos a consolidação da informação, do monitoramento e da avaliação como elementos que, além de possibilitar o aprimoramento da gestão, dos serviços, programas, projetos e benefícios, pudessem contribuir para o controle social da sociedade sobre o Estado. Estes elementos devem estar articulados entre si e ainda entre os entes federados, ou seja, seguem normas gerais do governo federal, mas Estados e Municípios podem e devem elaborar seus próprios instrumentos, conforme as particularidades locais. A NOB/SUAS de 2005 estabelece entre as responsabilidades dos Estados o apoio técnico aos municípios na estruturação e implantação de seus sistemas municipais de assistência social e, ainda, a instalação e coordenação de sistema estadual de monitoramento e avaliação das ações da assistência social, de âmbito estadual e regional, por nível de proteção básica e especial, em articulação com os sistemas municipais e validados pelo sistema federal. Os municípios, por sua vez, habilitados na gestão básica e plena do SUAS, também assumem a responsabilidade de instituir um plano de acompanhamento, monitoramento e avaliação. Segundo o documento os municípios devem: "instituir plano de acompanhamento, monitoramento e avaliação das ações de proteção social na rede própria e na rede prestadora de serviços, em articulação com o sistema estadual e de acordo com o sistema federal, pautado nas diretrizes da PNAS/2004" (BRASIL, 2005, p. 104).

Assim, considerando que o SUAS é um sistema orgânico e articulado entre as três esferas do governo, a responsabilidade pela realização de seu monitoramento e avaliação é da união, estados e municípios. Contudo, pensar neste processo de gestão de modo que, ao mesmo tempo envolva os entes federativos, inclua todos os aspectos do SUAS e não gere sobreposições e contradições é um grande desafio. Sabe-se que, no modelo federativo brasileiro, estados e municípios são entes com autonomia política e administrativa e, além disso, possuem competências comuns. Contudo, a articulação entre competências comuns e a necessidade de uma coordenação mínima exige políticas articuladas e com definição clara das responsabilidades de cada ente federado. No que se refere ao monitoramento e avaliação da política de assistência social há ainda uma indefinição quanto ao papel de cada ente federado na sua execução. Neste sentido, o governo federal privilegia o monitoramento e a avaliação dos serviços, programas e projetos cofinanciados, os estados privilegiam os seus programas de governo e os municípios ainda possuem muitas dificuldade técnicas e políticas para elaborar seus próprios planos de monitoramento e avaliação. Estes instrumentos de gestão são destinados, predominantemente, aos programas que utilizam os maiores volumes de recursos públicos e/ou possuem grande visibilidade política. Com isso, a avaliação e o monitoramento na área da assistência social ainda permanecem muito restritos a medição da eficiência da utilização dos recursos, isto é, voltados aos aspectos quantitativos de recursos e atendimentos, em detrimento de uma análise do impacto das ações na qualidade de vida da população destinatária da política.

Uma definição clara de cada instrumento de gestão pode contribuir para a superação desse impasse na gestão do SUAS. A recente publicação da NOB/SUAS/2012 descreveu cada um deles. No que se refere ao planejamento prevê o aperfeiçoamento da estrutura, descrição conceitual e de conteúdos mínimos dos Planos de Assistência Social, sendo que a sua elaboração deverá ser quadrienal coincidindo com os Planos Plurianuais de cada ente federado e, ainda precedido da realização de diagnóstico sócio territorial. A NOB/2012 também descreve a estrutura dos Planos que devem ter, entre outras, diagnóstico sócio territorial, metas estabelecidas, resultados e impactos esperados, indicadores de monitoramento e avaliação e espaço temporal de execução. Com isso, articula os instrumentos de planejamento, monitoramento e avaliação, posicionando-os com estratégias fundamentais para o aprimoramento da gestão e a qualificação dos serviços e benefícios. O monitoramento, 
por sua vez, é considerado uma função "inerente à gestão e ao controle social, e consiste no acompanhamento contínuo e sistemático do desenvolvimento dos serviços, programas, projetos e benefícios sócio assistenciais, em relação ao cumprimento de seus objetivos e metas" (BRASIL, 2012). É realizado por meio de indicadores e informações produzidas in loco, dados de sistemas de informação e dados de sistemas de informações específicos para a realização de monitoramento, sendo, portanto, uma responsabilidade da união, estados e municípios. No texto da NOB/SUAS/2012, também consta que o conjunto de indicadores do SUAS deve permitir acompanhar: a qualidade e o volume de oferta dos serviços, programas, projetos e benefícios de proteção social básica e proteção social especial; o cumprimento do Protocolo de Gestão Integrada de Serviços, Benefícios e Transferência de Renda; o desempenho da gestão de cada ente federativo e, o monitoramento do funcionamento dos Conselhos de Assistência Social e das Comissões Intergestores.

Diante disso, devemos considerar que o monitoramento é uma função inerente à gestão e deve ser realizada pela própria equipe de implementação do programa. Portanto, na gestão descentralizada da assistência social, esta responsabilidade torna-se maior para os municípios, os quais devem executar os serviços, programas, projetos e gerenciar benefícios, por ser o ente federado mais próximo da população. Em vista disso, os municípios precisam de capacidade institucional e gerencial, técnica, política, estruturas físicas, recursos humanos, além de orçamento, para cumprir todas as suas

... a avaliação e o

monitoramento na área da

assistência social ainda

permanecem muito restritos a

medição da eficiência da

utilização dos recursos, isto é, voltados aos aspectos

quantitativos de recursos e

atendimentos, em detrimento

de uma análise do impacto das

ações na qualidade de vida da

população ... responsabilidades enquanto ente federado autônomo. Entretanto, conforme demonstra Arretche (2000), a maioria dos municípios brasileiros é de pequeno porte populacional, possui densidade econômica pouco expressiva e significativa dependência de transferências fiscais. Por isso, os Estados também precisam contribuir decisivamente para compensar os obstáculos causados pelas dificuldades econômicas e fiscais dos municípios, e ainda as dificuldades técnicas de gestão das políticas sociais. Estes fatores determinam os diferentes graus de descentralização das políticas sociais, em especial, a política de assistência social.

No que se refere à avaliação, a mesma poderá ser realizada através de "contratação de serviços de órgãos e instituições de pesquisa, visando à produção de conhecimentos sobre a política e o sistema de assistência social" (BRASIL, 2012). Neste quesito, cabe à União a maior responsabilidade com as seguintes ações: promover continuamente avaliações externas de âmbito nacional abordando a gestão, os serviços, os programas, os projetos e os benefícios sócio assistenciais; estabelecer parcerias com órgãos e instituições federais de pesquisa visando à produção de conhecimentos sobre a política e o SUAS; realizar, em intervalos bianuais, pesquisa amostral de abrangência nacional com usuários do SUAS para avaliar aspectos objetivos e subjeti-

vos referentes à qualidade dos serviços prestados. Enquanto os Estados “poderão realizar avaliações periódicas da gestão, dos serviços e dos benefícios sócio assistenciais em seu território, visando subsidiar a elaboração e o acompanhamento dos planos" (BRASIL, 2012). Os municípios poderão "instituir práticas participativas de avaliação da gestão e dos serviços da rede sócio assistencial, envolvendo trabalhadores, usuários e instâncias de controle social" (BRASIL, 2012).

Segundo Costa (2007), apesar do consenso sobre a importância da avaliação, de fato avaliam-se muito pouco as ações na área da assistência social e, principalmente, há dúvidas se os resultados das avaliações são utilizados de maneira produtiva. $\mathrm{O}$ autor cita alguns fatores que contribuem para isso: a) existem poucos incentivos para a avaliação, pois o estilo de gestão predominante nas políticas sociais é pouco orientado para resultados; b) os objetivos geralmente são formulados de maneira genérica e ambiciosa para serem operacionais, ou seja, para orientarem as ações e a avaliação; c) gestores e instituições públicas são mais cobrados por planos de trabalho do que por resultados e inovações efetivamente obtidos; d) os processos de decisão no campo da assistência social tendem a ser frequentemente informais e pouco estruturados, o que leva a decisões pouco estratégicas, fragmentadas e reativas.

Diante destes fatores, Costa (2007) afirma que para a avaliação ser possível e relevante é preciso modificar a maneira como os projetos são formulados, ou seja, formular objetivos operacionais e realistas e explicar a teoria do projeto (por que e como se supõe que os insumos produzam resultados esperados); estruturar 
a tomada de decisão, criando-se espaços e momentos institucionalizados de reflexão sobre os resultados das avaliações adotando-se o planejamento estratégico; criar condições institucionais - financeiros e outros - para a busca de resultados para promover e utilizar a avaliação. Além disso, destaca que o sistema de monitoramento e avaliação deve estar orientado aos diferentes atores envolvidos na organização, não apenas aos tomadores de decisão, para estruturar o debate e a participação.

A avaliação participativa torna-se mais viável em âmbito municipal, onde se concretizam as ações da política de assistência social. Segundo Barreira (2000, p. 93), a avaliação de programas na assistência social deve investir necessariamente na capacitação emancipatória dos usuários, tendo em vista que "o referencial avaliativo na área volta-se para atendimento das necessidades e exigências das populações vulnerabilizadas pela pobreza e pela exclusão das redes de seguridade social". Por isso, a autora indica a avaliação com características participativas e de cunho emancipatório, para seguir os objetivos de inclusão social de seu público-alvo. A avaliação participativa inclui todos os sujeitos envolvidos na operacionalização do SUAS ou, no caso específico dos serviços, os profissionais e os usuários. A riqueza das discussões está no fato de que cada sujeito possui um olhar diferenciado para a mesma ação dependendo do lugar em que se situa. Entretanto, para sua realização são necessárias informações qualificadas e relevantes.

A informação, imprescindível, para a realização do monitoramento e avaliação também ganhou destaque com a NOB/SUAS/2012, a partir da definição de diretrizes para a concepção dos sistemas de informação no SUAS, sendo elas: compartilhamento da informação na esfera federal, estadual e municipal e entre todos os atores do SUAS, trabalhadores, conselheiros, usuários e entidades; compreensão de que a informação no SUAS não se resume à informatização ou instalação de aplicativos e ferramentas, mas afirma-se também como uma cultura a ser disseminada na gestão e no controle social; disponibilização da informação de maneira compreensível à população com transparência e acessibilidade; construção de aplicativos e subsistemas flexíveis que respeitem as diversidades e particularidades regionais; e interconectividade entre os sistemas.

Desde a criação do Ministério de Desenvolvimento Social e Combate à Fome (MDS), em 2004, a informação, o monitoramento e a avaliação são institucionalizados a partir da criação de uma secretaria específica para este fim, representando grande avanço na área e o incentivo a Estados e municípios para incorporarem estes instrumentos também em seu âmbito de atuação. A Secretaria de Avaliação e Gestão da Informação (SAGI) foi colocada no mesmo patamar das outras secretarias do MDS: Secretaria Nacional de Renda e Cidadania (SENARC), Secretaria Nacional de Segurança Alimentar e Nutricional (SESAN), Secretaria Nacional de Assistência Social (SNAS), e Secretaria de Articulação para Inclusão Produtiva (SAIP).

Para Vaitsman e Paes-Sousa (2011, p. 23), a institucionalização da avaliação na assistência social significou maior profissionalização da gestão, porém reflete os tradicionais conflitos entre as esferas técnicas e políticas, uma vez que a avaliação de políticas e programas públicos é "uma atividade exercida em um terreno atravessado pela política, onde os estudos extrapolam o simples âmbito da produção do conhecimento". Afirmam ainda que embora seja uma pesquisa aplicada às políticas públicas, fazem parte das disputas políticas do setor avaliado e da política pública de modo geral. O processo de institucionalização da avaliação na área da assistência social em âmbito federal dependeu de um conjunto de condições como, por exemplo, a decisão política de implantar um sistema de avaliação com alocação de recursos financeiros, físicos e humanos e, ainda, a cooperação entre os membros da organização. Assim, para a institucionalização da avaliação foi necessário o compartilhamento de dados gerenciais sobre os programas, dados físico-financeiros, informações, conhecimentos e contatos dos municípios (VAITSMAN; PAES-SOUSA, 2011).

A seguir faremos alguns comentários sobre os desafios à implementação de sistemas de avaliação e monitoramento na política de assistência social a partir de dados coletados em municípios de pequeno porte do Estado do Paraná, foco de nossa pesquisa.

\section{Desafios do processo de avaliação e monitoramento em municípios de pequeno porte}

No Estado do Paraná, entre 2009 e 2010, foi realizado um processo piloto denominado Avaliação, Monitoramento e Habilitação Municipal que atingiu todos os municípios paranaenses. Este processo foi elaborado pela extinta Secretaria de Estado do Trabalho, Emprego e Promoção Social (SETP), responsável pela coordenação da política de assistência social no Estado entre 2003 e 2010, e executado em três fases. A primeira foi a análise dos instrumentos de gestão - Plano Municipal de Assistência Social e Relatório de Gestão - instrumentos de planejamento e de avaliação desta política em âmbito municipal. A segunda fase foi à visita in loco dos técnicos dos Escritórios Regionais (E.R/SETP) aos municípios para o preenchimento de questionário referente à gestão municipal, com a finalidade de gerar um plano de providências com indicações de prazos e responsáveis para os reordenamentos de ações na perspectiva de aprimoramento da gestão municipal do 
Sistema Único de Assistência Social (SUAS). Na terceira fase, os municípios reuniram (ou não) documentos que comprovassem o cumprimento do plano de providências, ou seja, que haviam reordenado as ações em conformidade com as orientações finais do processo de monitoramento e avaliação.

Realizamos estudo qualitativo sobre esse processo na região centro-oeste do Estado do Paraná, região de Guarapuava, composta atualmente por dez municípios. A escolha desta região ocorreu porque pesquisas anteriores demonstraram as dificuldades na gestão da política de assistência social dos municípios e, principalmente, os entraves nas tentativas de estabelecer sistemas de monitoramento e avaliação. Ademais, acreditávamos que a região de Guarapuava nos daria uma amostra do processo que ocorreu em todo o Estado paranaense devido a sua localização geográfica e sua composição enquanto região, formada por um município de grande porte, dois municípios de pequeno porte 02 e sete municípios de pequeno porte 01 . Os procedimentos de coleta de dados envolveram pesquisa documental nos registros do processo de monitoramento, avaliação e habilitação municipal disponíveis no escritório regional da extinta SETP, em Guarapuava, e entrevistas semiestruturadas com profissionais que participaram ativamente do processo, tanto em âmbito estadual, quanto municipal. No âmbito estadual foram selecionadas: uma profissional da SETP/sede e duas profissionais do escritório regional de Guarapuava. No nível municipal foram: três secretárias municipais de assistência social e cinco profissionais municipais responsáveis pelo gerenciamento da política de assistência social.

A partir da análise documental e das entrevistas destacaremos as considerações finais do estudo, a partir de três observações. A primeira refere-se à importância do nível estadual na implementação da política de assistência social na perspectiva do SUAS. O fato do governo federal se relacionar diretamente com o governo municipal e, este último, ter papel principal na implementação dos serviços devido a proximidade com a população, tem praticamente gerado uma ausência do Estado na gestão da política. Porém, quando o Estado assume o seu papel ativo na assessoria aos municípios, ele modifica esta relação e contribuiu para o avanço significativo na organização da assistência social.

A segunda observação é que o processo de monitoramento, avaliação e habilitação municipal realizado no Estado do Paraná, entre 2009 e 2010, contribuiu para o aprimoramento da gestão da assistência social no Estado na medida em que apontou as fragilidades e indicou os caminhos para a sua superação. Além disso, envolveu as instâncias participativas e deliberativas da política e vinculou o processo de monitoramento com o avanço no nível de gestão do SUAS, o que possibilitou o avanço da organização da gestão nos municípios.

O processo de monitoramento apresentou algumas fragilidades: o número reduzido de recursos humanos da gestão estadual; os entraves políticos que influenciaram na sua descontinuidade, ou seja, a mudança do governo Estadual que interrompeu o processo na terceira fase, sem que houvesse uma avaliação e continuidade; as dificuldades de diálogo entre Estado e municípios, uma vez que as entrevistas realizadas com gestores da região de Guarapuava/Paraná demonstraram compreensões não muito claras sobre os objetivos do processo de monitoramento. Outras fragilidades detectadas são: a ausência de indicadores de monitoramento; a divulgação do resultado final restrita aos envolvidos no processo no nível estadual; o destaque a documentos comprobatórios; o fato de não ter sido previsto auxílio financeiro aos municípios para as adequações e, enfim, de não ter sido estabelecido o que fazer se a concepção da gestão municipal estivesse vinculada a visão tradicional da assistência social ou houvesse resistências nas adequações.

Outra questão que merece destaque é o fato de prevalecer a dimensão técnica-operativa da política, que privilegia uma análise da implementação da legislação vigente, conforme o previsto, o que muitas vezes reforça traços conservadores. É preciso avançar na análise dos impactos e efeitos dos serviços sócio assistenciais na vida da população beneficiária e na análise do alcance dos objetivos da política.

Por fim, a terceira observação refere-se especificamente à região de Guarapuava/Paraná. Apesar dos dados socioeconômicos demonstrarem a necessidade de estruturação de uma rede de proteção social e, em particular, da organização da política de assistência social para quem dela necessitar, os municípios da região possuem inúmeras dificuldades técnicas, políticas e financeiras. Há dificuldades técnicas no estabelecimento de instrumentos de gestão - diagnósticos, planejamento, monitoramento e avaliação - articulados e integrados que possam ultrapassar as ações pontuais, improvisadas e imediatistas. Estes instrumentos, quando subordinados à sua dimensão política de produzir informações para subsidiar o controle social e a ampliação de direitos, podem contribuir para o fortalecimento da assistência social enquanto política.

Soma-se a estas dificuldades a grande rotatividade de profissionais ou o fato dos municípios de pequeno porte 01 , possuírem um reduzido número de profissionais lotados na gestão da política, o que foi apontado como sendo o maior desafio no Estado do Paraná. Além disso, 80\% dos secretários de assistência social possuem vínculo de parentesco com o prefeito. Os municípios ainda reproduzem o fenômeno do primeiro damismo na área. As dificuldades políticas assim ficam vinculadas a visão tradicional e moderna da Assistência Social que direcionam as ações no âmbito municipal. A visão ideopolítica pode contribuir para avanços, mas também para retrocessos na área. 
A avaliação ainda é percebida como opinião no sentido lato senso, ou seja, informal/espontânea carecendo de instrumentos de coleta e análise de dados advindos da pesquisa social aplicada. Já o monitoramento é vinculado à fiscalização, controle, vigilância, organização de documentos e ao cumprimento de normas em detrimento da sua valorização enquanto acompanhamento planejado, sistemático e dirigido da implementação de um serviço, programa ou projeto social. A informação e os sistemas informatizados ainda não são considerados como instrumento de organização e sistematização de dados que servem para subsidiar processos de planejamento, mas como uma forma de controle de benefícios eventuais.

As dificuldades financeiras na política de assistência social advêm, de um lado, do próprio município, que direciona poucos recursos para a área e, de outro lado, da falta de financiamento a fundo do Estado, que permanece com repasses focalizados via convênios. $\mathrm{O}$ governo federal tem avançado na discussão sobre o financiamento da gestão e dos serviços na área, porém ainda faltam regulamentações e a concretização das previsões da Norma Operacional Básica do Sistema Único de Assistência Social - NOBB/SUAS/2012 (ALBUQUERQUE, et al., 2012), que prevê modalidades de financiamento via blocos, conforme os níveis de proteção básica e especial e a gestão do SUAS, o que, acredita-se, poderá possibilitar maior autonomia para os municípios.

\section{Considerações finais}

No Brasil, a incorporação da função avaliação nas políticas sociais se deu, principalmente, a partir da década de 1990, devido à discussão sobre a necessidade de modernização da administração pública em uma perspectiva gerencial. A avaliação é considerada um instrumento de gestão que garante eficiência, eficácia e efetividade às ações do Estado, tendo como modelo as empresas privadas. Assim a ênfase maior é na sua dimensão técnica que escamoteia a sua dimensão política, a qual se refere aos motivos que levaram à sua realização e não realização, utilização e não utilização e, ainda, divulgação e não divulgação dos resultados.

Destaca-se que a institucionalização da avaliação na área da assistência social é parte de um processo mais amplo de construção da democracia e da profissionalização da administração pública, uma vez que a avaliação e o monitoramento, ainda que representem a necessidade de estabelecimento de mecanismos gerenciais, respondem também à pressão da sociedade por transparência e responsabilização da gestão pública.

Em relação ao processo de monitoramento desenvolvido no Paraná, apesar das fragilidades, foi importante porque possibilitou um olhar atento dos profissionais, tanto estaduais quanto municipais, à organização da assistência social. Os instrumentos de gestão, em especial o monitoramento e a avaliação, se forem pensados em sua dimensão política, conforme prevê a legislação na área, podem ser impulsionadores do avanço da assistência social como política pública profissionalizada e qualificada.

Por fim, reforçamos, nas três esferas de governo, a necessidade do debate coletivo sobre a necessidade do estabelecimento de uma cultura de avaliação e monitoramento da política de assistência social.

\section{Referências}

AlbuQUerQue, S. A.; GOMES, W. A. A.; TRIVElinO, A. De S. Norma Operacional Básica do Sistema Único de Assistência Social. In: Revista do Fórum Nacional de Secretários (as) de Assistência Social. n.1, dez. 2012, p. 93-97.

ARRETCHE, M. T. da S. Estado federativo e políticas sociais: determinantes da descentralização. Rio de Janeiro: Revan, São Paulo: FAPESP, 2000.

BARREIRA, M. C. R. N. Avaliação participativa de programas sociais. São Paulo: Veras; Lisboa: CPIHTS, 2000.

BRASIL. MDS; CNAS. NOB - Norma Operacional Básica: Resolução n 33, de 12 de dezembro de 2012. Brasília: MDS, 2012.

. MDS; CNAS. NOB - Norma Operacional Básica: Resolução nº 130, de 15 de julho de 2005. Brasília: MDS, 2005.

. MDS; CNAS. PNAS - Política Nacional de Assistência Social: Resolução nº 145, de 15 de outubro de 2004. Brasília: MDS, 2004.

COSTA, B. L. D. A avaliação nas políticas de assistência social: desafios para sua consolidação e para sua relevância. In: FAHEL, M.; NEVES, J. A. B. (Orgs.). Gestão e avaliação de políticas sociais no Brasil. Belo Horizonte: PUC Minas, 2007, p. 193-215.

FAGUNDES, H.; MOURA, A. B. de. Avaliação de programas e políticas públicas. Revista Textos \& Contextos, v. 8, n.1, Porto Alegre, jan./jun. 2009, p. 89-103.

GOMES, M. de F. C. M. Avaliação de políticas sociais e cidadania: pela ultrapassagem do modelo funcionalista clássico. In: SILVA, M. O. da S. (Org.). Avaliação de políticas e programas sociais: teoria e prática. São Paulo: Veras, 2001, p. 17-36.

JANNUZZI, P. de M. Monitoramento analítico como ferramenta para aprimoramento da gestão de programas sociais. Revista da Rede Brasileira de Monitoramento e Avaliação, n. 1, jan.jun., 2011, p. 36-65.

JOPPERT, M. P. Pesquisa sobre Oferta e Demanda por Serviços de Avaliação no Setor Público: o caso brasileiro. Março de 2011. Disponível em: <http://api.ning.com.br〉. Acesso em: 13 jul. 2013. 
LOBO, T. Avaliação de processos e impactos em programas sociais: algumas questões para reflexão. In: RICO, E. M. (Org.). Avaliação de Políticas Sociais: uma questão em debate. São Paulo: Cortez: Instituto de Estudos Especiais, 2009, p. 75-84.

MELO, M. A. As sete vidas da agenda pública brasileira. In: RICO, E. M. (Org.). Avaliação de Políticas Sociais: uma questão em debate. São Paulo: Cortez: Instituto de Estudos Especiais, 2009, p. 11-28.

VAITSMAN, J.; PAES-SOUSA, R. Avaliação de programas e profissionalização da gestão pública. Revista da Rede Brasileira de Monitoramento e Avaliação, n. 1, jan./jun. 2011, p. 21-37.

\section{Nayara Cristina Bueno}

nayara_cbo@hotmail.com

Mestre em Serviço Social pela Universidade Estadual de Londrina (UEL)

Professora do curso de Serviço Social da Universidade Estadual do Paraná (UNESPAR)

\section{UNESPAR}

Campus de Paranavaí

Av. Gabriel Esperidião, SN

Paranavaí - Paraná - Brasil

CEP: 87703-000

\section{Cássia Maria Carloto}

cmcarloto@gmail.com

Doutorado e Pós-doutorado em Serviço Social pela Pontifícia Universidade Católica de São Paulo (PUC/SP) Professora do curso de Serviço Social da Universidade Estadual de Londrina (UEL)

\section{UEL}

Rodovia Celso Garcia Cid, PR 445, Km 380

Campus Universitário

Londrina - Paraná - Brasil

CEP: 86057-970 